\title{
Preponderance of cells with stem cell characteristics in metastasising mouse mammary tumours induced by deregulated EphB4 and ephrin-B2 expression
}

\author{
PHILIP KAENEL*, CAROLINE SCHWAB*, KATHRIN MÜLCHI*, \\ CARLOS WOTZKOW and ANNE-CATHERINE ANDRES \\ Department of Clinical Research, University of Bern, Tiefenaustrasse 120c, CH-3004 Bern, Switzerland
}

Received July 16, 2010; Accepted September 2, 2010

DOI: 10.3892/ijo_00000834

\begin{abstract}
We have previously shown that EphB4 and ephrin-B2 are differentially expressed in the mammary gland and that their deregulated expression in the mammary epithelium of transgenic mice leads to perturbations of the mammary parenchyma and vasculature. In addition, overexpression of EphB4 and expression of a truncated ephrin-B2 mutant, capable of receptor stimulation but incapable of reverse signalling, confers a metastasising phenotype on NeuT initiated mouse mammary tumours. We have taken advantage of this transgenic tumour model to compare stem cell characteristics between the non-metastasising and metastasising mammary tumours. We analysed the expression of the proliferation attenuating $\mathrm{p} 21^{\text {waf }}$ gene, which was significantly increased in the metastasising tumours. Moreover, we compared the expression of CK-19, Sca-1, CD24 and CD49f as markers for progenitor cells exhibiting a decreasing differentiation grade. Sca-1 expressing cells were the earliest progenitors detected in the non-metastasising NeuT induced tumours. The metastasising NeuT/EphB4 tumours were enriched in CD24 expressing cells, whereas the metastasising NeuT/truncated ephrin-B2 tumours contained in addition significant amounts of CD49f expressing cells. The same cell populations were also enriched in mammary glands of single transgenic MMTV-EphB4 and MMTV-truncated ephrin-B2 females indicating that deregulated EphB4-ephrin-B2 signalling interferes with the homeostasis of the stem/progenitor cell pool before tumour formation is initiated. Since the same cell populations are enriched in the normal tissue, primary mammary tumours and metastases we conclude that these progenitor cells were the origin of
\end{abstract}

Correspondence to: Dr Anne-Catherine Andres, Department of Clinical Research, Tiefenaustrasse 120c, CH-3004 Bern, Switzerland E-mail: anne-catherine.andres@dkf.unibe.ch

*Contributed equally

Key words: carcinogenesis, MMTV-NeuT, progenitor cells, transgenic mice, tumour initiating cells tumour formation and that this change in the tumour origin has led to the acquisition of the metastatic tumour phenotype.

\section{Introduction}

Carcinogenesis is a multi-step process involving sequential mutations affecting epithelial growth and leading eventually to malignant and metastasising tumours. Whereas the disease in the non-invasive in situ stage has a high probability of cure, invasive and metastasising tumours, in the long run, usually fail to respond to current treatment protocols and as a result metastatic breast cancer remains an incurable disease (1).

One of the first events in the transition from a benign to the highly malignant state is the ability of cells to overcome cell adhesion and attain mobility. This is achieved by epithelialmesenchymal transition, a process which is also instrumental during embryogenesis and in post-natal tissue remodelling as observed during the mammary gland life cycle (2). Furthermore, metastatic cells have to settle into a permissive environment which sustains their further growth, a process which has been summarized in the seed and soil hypothesis (3). Although some properties of metastatic cells are well characterized, the molecular mechanisms allowing detached and migrating cells to seed and survive, rather than to be eliminated by apoptosis or the immune system, are still obscure. Moreover, the fact that recurrence of primary or metastatic tumour growth can occur after decades of disease-free survival is a particular problem of breast cancer and the mechanisms underlying this tumour dormancy are still poorly understood (4).

Although metastasis formation is a common aspect of carcinogenic diseases, experimental evidence gained from leukemias and breast tumours suggests that in fact only a small subset of cancer cells have metastatic potential $(5,6)$. The observation that these cells exhibit stem cell characteristics led to the cancer stem cell hypothesis which implicates mutated stem cells or tumour cells with acquired stem cell characteristics in tumour recurrence and metastasis formation (7). Stem cells of normal tissues are slowly replicating cells which by asymmetric cell division give rise to two different daughter cells, one remaining a stem cell and the other giving rise to faster dividing precursor cells which evade the stem cell niche and regenerate the tissue. In parallel, tumour stem cells or tumour initiating cells are thought to resist proliferation 
dependent therapies, persist and may upon certain stimulation regenerate the tumour tissue (8). Thus, the concept of tumour dormancy in micro-metastases and the often decade-long latency of breast tumours could directly be related to the biology of mammary stem cells (9). Although of potential therapeutic importance, little is still known about the origin of mammary tumour initiating cells and their molecular control. Strikingly, many signalling pathways regulating the mammary stem population, such as the notch-, wnt- or bim-signalling, have also been implicated in breast carcinogenesis (10).

The large families of signalling molecules, Eph and ephrin, play a key role in the specification of cell fate, mobility, sorting and compartmentalisation of cell populations. Since both the Eph receptors and ephrin ligands are membrane-bound molecules, receptor-ligand interaction requires direct cell-cell contact and elicits forward signalling form the receptor and reverse signalling emanating from the ligand leading to bidirectional cell-cell communication. Members of the Ephephrin family, predominantly EphB4 and ephrin-B2, play a key role not only in the development of the embryonic vasculature but also in neo-vascularisation in the adult (11). In addition to angiogenesis, EphB4 and ephrin-B2 have been implicated in platelet clustering and in blood clotting at sites of vascular injury (12), hematopoiesis and migration of hematopoietic cells $(13,14)$, the control of bone homeostasis $(15)$ and mammary gland morphogenesis $(16,17)$. Interestingly, there is growing evidence that Ephs and ephrins are also involved in the control of stem cell compartments and of the asymmetric stem cell division in a variety of tissues (18-22).

Considering the key roles of Ephs and ephrins in normal homeostasis, it is not surprising that their deregulated activity is implicated in a variety of neoplastic disorders. Ephs and ephrins have been shown to play a role in tumour growth, metastasis formation and tumour angiogenesis (23). In particular, aberrant EphB4 and ephrinB2 expression, often upregulation, is associated with carcinogenesis in several human tumours (24-26), including breast cancer (27-30). It has been reported that EphB4 can promote tumour growth by stimulating angiogenesis through ephrin-B2 $(29,31)$. Despite these observations, the role(s) of EphB4 and ephrin-B2 in carcinogenesis are still unclear. Whereas EphB4 has been described as a survival factor in breast cancer (32), head and neck squamous carcinoma (33) and colorectal cancer $(34,35)$, others have presented evidence that EphB4 can serve as a tumour suppressor in breast (30) and colorectal cancer (36).

We have previously shown that EphB4 and ephrin-B2 are differentially expressed in the mammary gland and that deregulated EphB4 or ephrin-B2 expression in the mammary epithelium of transgenic mice leads to perturbations in growth and architecture of the mammary parenchyma and vasculature $(16,17,37)$. In addition, overexpression of EphB4 and expression of a dominant negative ephrin-B2 mutant, capable of receptor stimulation but incapable of reverse signalling, confers an invasive and metastasising phenotype on NeuT initiated non-invasive mouse mammary tumours $(16,17)$. In this report, we have taken advantage of this transgenic tumour model to compare the characteristics of the nonmetastasising and metastasising mammary tumours. Our results indicate that the metastasising phenotype induced by
EphB4 and mutant ephrin-B2 is characterized by an increase of tumour cells with stem/progenitor cell characteristics. We further present evidence that overexpression of EphB4 and inhibition of ephrin-B2 reverse signalling directly affect the mammary stem/progenitor cell population thereby increasing the cell population prone to mutate to tumour stem cells.

\section{Materials and methods}

Transgenic lines and tumour specimens. The establishment of the transgenic lines has been described previously. Briefly, for the EphB4 overexpressing mice, the full length murine EphB4 cDNA has been placed under the control of the MMTV-LTR promoter and the MMTV-EphB4 transgenic mice were established by pronuclear injection (16). For the dominant negative ephrin-B2 mutant mice a fragment encoding the intracellular part encoding the conserved tyrosine residues was removed from the murine ephrin-B2 cDNA, leaving the sequences encoding the transmembrane region and the extracellular part intact. Thus, the encoded protein exhibits the correct membrane localization, is able to interact with EphB4 and to dimerize with endogenous ephrin-B2 which leads to stimulation of receptor forward signalling, but abolishes ephrin-B2 derived reverse signalling. The construct was placed under the control of the MMTV-LTR promoter and the MMTV-truncated ephrin-B2 transgenic mice were established by pronuclear injection (17). Transgenic mice were crossed over 6 generations with inbred C57/B16 mice (Charles River, Wiga, Sulzfeld, Germany) in order to reduce complexity of the genetic background. MMTV-LTR-NeuT transgenic mice of the FBV strain were obtained commercially (Charles River). Tumor development was followed in the F1 generation of FBV x C57/B16 crosses. Of each group, 10 tumour specimens and for the metastasising groups 4 metastases were included in this study. All animal studies were conducted according to the institutional ethics guidelines.

Immunohistochemistry. Tumour-bearing animals were injected with $200 \mu \mathrm{g}$ per g body weight of bromodeoxyuridine (BrdU, Sigma, Buochs, Switzerland) in PBS $3 \mathrm{~h}$ before sacrifice. Tumours were removed and fixed in $4 \%$ paraformaldehyde for $4 \mathrm{~h}$. Tissue preparation and immunohistochemistry was essentially done as described except that incubation with the primary antibodies was routinely done overnight at $4^{\circ} \mathrm{C}$ (37). Antisera were obtained commercially: anti-BrdU (Roche Diagnostics, Rotkreuz, Switzerland), anti CD24 and antiCD49f (AbDserotec, Kidlington, UK), CK-19 (Santa Cruz Biotechnology, Santa Cruz, CA), anti-ER $\alpha$ (Novocastra, Newcastle upon Tyne, UK), anti-p21 (Santa Cruz Biotechnology), anti-Sca-1 (BD Biosciences, San Jose, CA). Epitope retrieval was done by microwaving as described for anti-BrdU, -CK19 , -ER $\alpha,-p 21$ or by Proteinase $\mathrm{K}$ digestion for $20 \mathrm{~min}$ at $37^{\circ} \mathrm{C}$ for anti-CD24, -CD49f, Sca-1.

Quantitative evaluation. For quantitative evaluation 500 cells were counted in each of three arbitrarily chosen visual fields and the number of positive cells therein was determined. Values are given as percentage of positive cells. For the mitotic index, mitotic figures were counted in three visual fields at a x20 magnification. Statistical significance was calculated using the Student's t-test. 

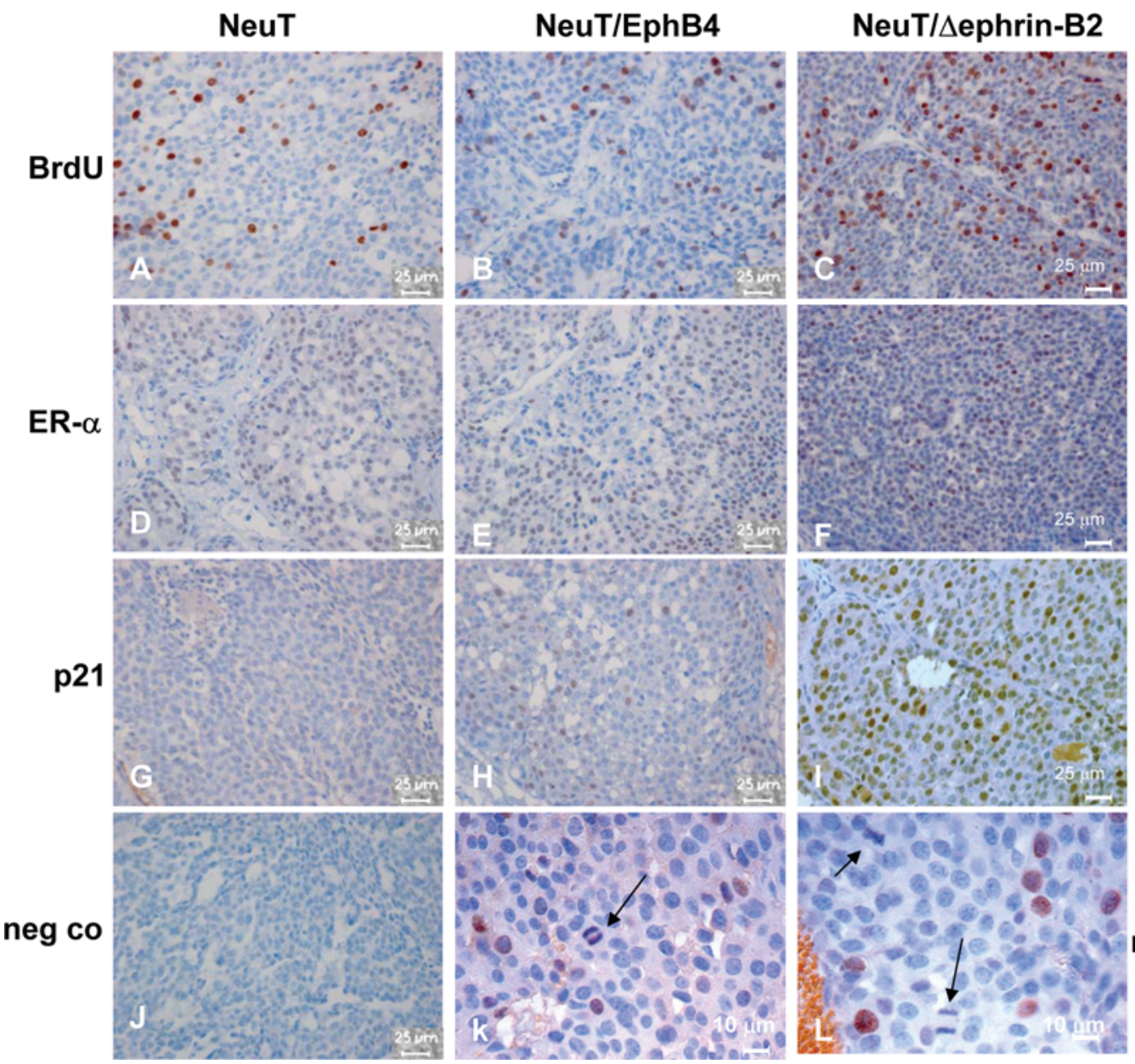

mitotic figures

Figure 1. Expression of cell proliferation-related proteins. Immunohistochemical detection of BrdU incorporation (A-C), estrogen receptor- $\alpha$ (ER $\alpha$ ) expression (D-F), and p21 waf (p21) expression (G-I) in NeuT (A, D, G), NeuT/EphB4 (B, E, H) and NeuT/truncated ephrin-B2 (Lephrin-B2) (C, F, I) induced tumours. Mitotic figures are frequently seen in NeuT/EphB4 (K) and NeuT/truncated ephrin-B2 (L) induced tumours. K and L are taken from the detection of BrdU incorporation, note that mitotic figures are either BrdU positive (K) or negative (L). J, negative control omitting the first antibody.

FACS analysis. For FACS analysis the no. 4 mammary glands were dissected from resting adult female mice. Six agematched females of each line were analysed. After mechanical dissection using scalpels, the tissue was transferred to enzymatic digestion in complete EpiCult ${ }^{\circledR}-\mathrm{B}$ medium (StemCell Technologies, Grenoble, FR) supplemented with collagenase/ hyaluronidase (StemCell Technologies) and 5\% fetal calf serum. Generation of a single cell suspension for antibody staining was performed according to the protocol of the manufacturer (StemCell Technologies).

The antibodies used for flow cytometry were purchased from Biolegend (San Diego, CA) and included: biotinylated TER119 (clone TER-119), biotinylated CD31 (clone MEC13.3), biotinylated CD45 (clone 30-F11), Streptavidin-PE/Cy7, CD49f-FITC (clone GoH3) and CD24-PE (clone M1/69). Isotype controls were performed with the corresponding mouse $\mathrm{IgG} 1 \kappa$ isotype Control antibodies from Biolegend. Compensation was performed using the $\mathrm{BD}^{\mathrm{TM}}$ CompBeads Compensation Particles Set (BD Biosciences). For staining, cells were incubated at a density of 5-10x $10^{5}$ cells $/ \mathrm{ml}$ in PBS, $2.5 \%$ fetal calf serum, $10 \mathrm{mM}$ EDTA, $0.2 \%(\mathrm{w} / \mathrm{v})$ sodium azide containing the antibodies for $25 \mathrm{~min}$ at $4^{\circ} \mathrm{C}$ with the primary antibodies and subsequently $15 \mathrm{~min}$ at $4^{\circ} \mathrm{C}$ with the secondary antibodies. Analysis was carried out with FACSDiva software on a BD ${ }^{\mathrm{TM}}$ LSRII (BD Biosciences). Samples were primarily gated on forward- and side-scatter. Contaminating endothelial and haematopoietic cells were excluded by gating for CD31, CD45 and TER119 negative cells and the lineage negative cells finally analysed for CD24 and CD49f expression.

\section{Results}

In order to elucidate the function of EphB4 and ephrin-B2 in mammary gland biology, we previously established transgenic mice overexpressing EphB4 or ephrin-B2 as well as a dominant negative ephrin-B2 mutant (truncated ephrin-B2) which is capable inducing receptor signalling but is unable to elicit the ligand derived reverse signalling. To date, mammary tumours have not been observed in the three transgenic mouse lines, indicating that deregulated EphB4 or ephrin-B2 expression per se is not sufficient for malignant transformation of mammary epithelial cells. We have, however, crossed these transgenic mice with transgenic mice overexpressing the NeuT oncogene in the mammary gland. These experiments, summarized in Table I, have revealed that overexpression of EphB4 or of truncated ephrin-B2 are capable of specifically altering the tumour cells to the metastatic phenotype $(16,17)$. Thus, these transgenic mice represent 
Table I. Incidence of primary mammary tumours and lung metastases in the different transgenic lines.

\begin{tabular}{lcc}
\hline Transgenic line & $\begin{array}{c}\text { Incidence of } \\
\text { primary tumours } \\
(\%)\end{array}$ & $\begin{array}{c}\text { Incidence of } \\
\text { lung metastasis } \\
(\%)\end{array}$ \\
\hline NeuT & 100 & 0 \\
NeuT/EphB4 & 92 & 86 \\
NeuT/truncated & 90 & 92 \\
ephrinB & & \\
\hline
\end{tabular}

promising mouse models for the direct characterisation of and comparison between the non-metastatic and metastatic phenotype of mammary cancer cells and allow the investigation of the alterations induced by the deregulated EphB4 and ephrin-B2 expression.

Analysis of cell proliferation parameters. In order to compare the growth rate of the different tumour types we have analysed cell proliferation by immunohistochemical detection of BrdU incorporation. In all tumours proliferating cells were readily detected (Fig. 1A-C). Determination of the percentage of proliferating cells revealed that the proliferation rate, ranging between 9 and $13 \%$, did not differ significantly between the non-metastasising and metastasising tumours $(\mathrm{p}=0.48$ and 0.27 ) (Fig. 2A).
Since estrogen is a main inducer of mammary epithelial proliferation we next analyzed the presence and localization of activated estrogen receptor- $\alpha$ (ER- $\alpha)$. In all tumour samples analyzed nuclear localization of ER- $\alpha$, indicative for the activated form of the receptor, was evident in $35-45 \%$ of the tumour cells (Fig. 1D-F). Similar to the proliferation rate, the percentage of ER- $\alpha$ positive cells did not differ significantly between the different tumour types ( $\mathrm{p}=0.19$ and 0.07) (Fig. 2B). These results indicate that all tumours exhibit a moderate growth rate which may at least in part be estrogen-dependent.

The tumour suppressor $\mathrm{p} 21^{\text {waf }}$ is a negative regulator of the cell cycle and its expression has been associated with the maintenance of progenitor cell pool in mammary gland and skin (38). Since p21 waf is also likely to be involved in the attenuation of tumour stem cell division we have immunohistochemically analysed $\mathrm{p} 21^{\text {waf }}$ expression in our tumour models. p21 ${ }^{\text {waf }}$ positive cells were almost undetectable in the NeuT induced tumours (Fig. 1G). In contrast, the metastasising double transgenic tumours contained $\mathrm{p} 21^{\text {waf }}$ positive cells regularly interspersed throughout the tumour tissue (Fig. 1H and I). The quantitative analysis revealed the significant difference in the number of these putative slow dividing cells. Whereas only $1 \%$ of NeuT induced tumour cells expressed p $21^{\text {waf }}$, both the NeuT/EphB4 and the NeuT/ephrin-B2 induced tumours contained with 10 and $11 \%$, resp., significantly more p21 waf positive cells $(p<0.0001$ for both lines). Thus, although the percentage of proliferating cells was similar in all tumour types, the metastasising tumours exhibited a significantly higher number of slow or non-dividing cells. Interestingly, in these tumours mitotic figures were regularly seen (Fig. $1 \mathrm{~K}$ and L)

\section{A}
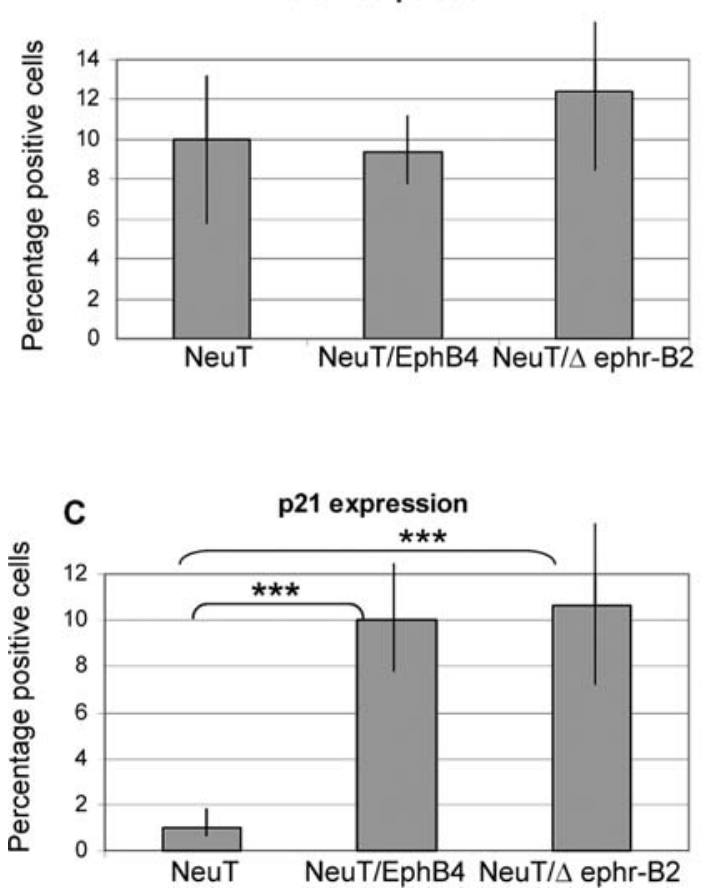

B
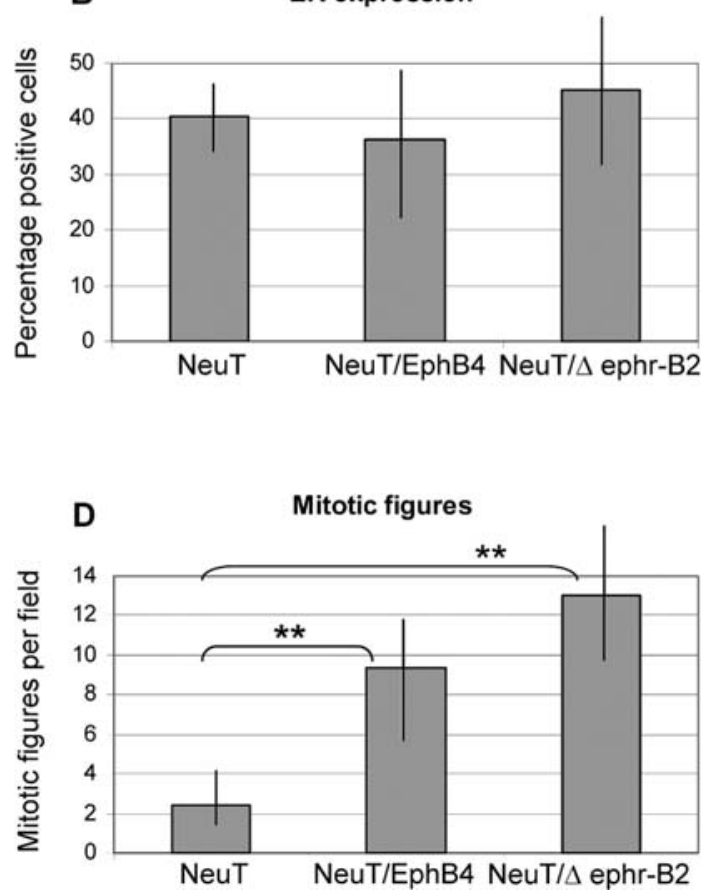

Figure 2. Quantification of BrdU incorporation (A), estrogen-receptor- $\alpha$ (ER $\alpha$ ) expression (B), p12 waf expression (C) and the mitotic index (D) in the NeuT, NeuT/EphB4 and NeuT/truncated ephrin-B2 ( $\Delta$ ephrin-B2) induced tumours. Bars represent standard deviation. ${ }^{* *} \mathrm{p}<0.01,{ }^{* * *} \mathrm{p}<0.001$. 


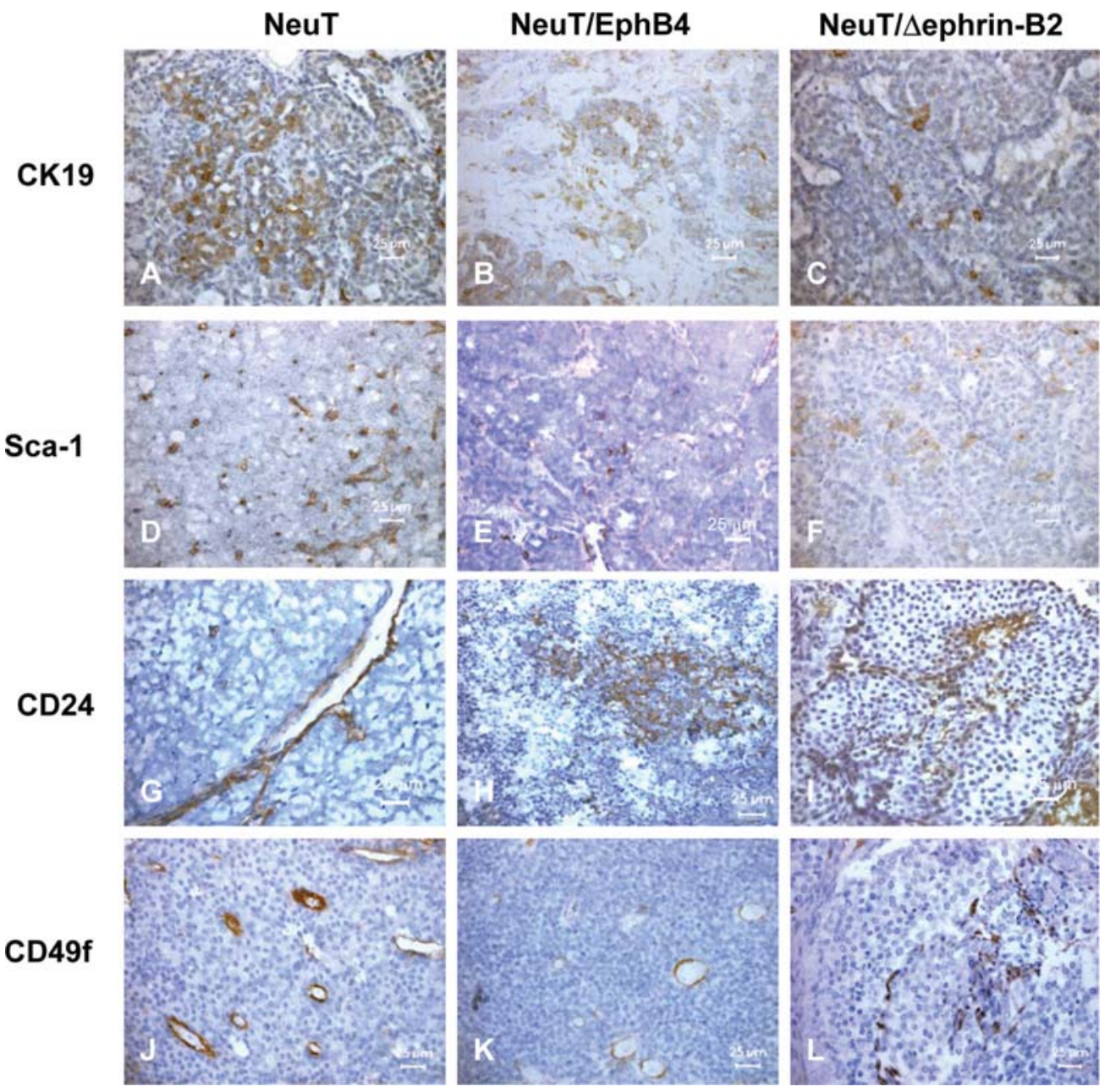

Figure 3. Expression of stem/progenitor cell markers. Immunohistochemical detection of cytokeratin-19 (CK19) (A-C), stem cell antigen-1 (Sca-1) (D-F), CD24 (G-I) and CD49f (J-L) expression in NeuT (A, D, G, J), NeuT/EphB4 (B, E, H, K) and NeuT/truncated ephrin-B2 ( $\Delta$ ephrin-B2) (C, F, I, L) induced tumours.

and indeed, the mitotic index was significantly increased from 2 for NeuT induced tumours to 9.5 and $13 \%$ in the doubletransgenic tumours ( $\mathrm{p}=0.009$ and 0.005$)$ (Fig. 2D). In summary we can conclude that in this model, metastasising tumours contain an increased population of putative slow dividing cells, however, the remaining proliferating cells seem to exhibit a faster progression through the cell cycle.

Detection of potential tumour stem/progenitor cells. Considering that tumour stem cells are thought to be responsible for metastasis formation and that we observed an increase in division-competent but attenuated cells in the metastasising tumours, we analyzed the different tumour types for the presence of cells with stem cell properties. We have analyzed the expression of the following marker proteins indicative for a decreasing differentiation grade: cytokeratin-19 (CK19) indicative for epithelial cells before end-differentiation, stem cell antigen-1 (Sca-1) indicative for ER-positive progenitor cells, CD24 indicative for luminal restricted progenitor cells and finally CD49f indicative for the unrestricted and bi-potent mammary stem/progenitor cells (39).

The immunohistochemical detection of CK-19 expression revealed that most of the tumour cells lost CK-19 expression.
CK-19 positive cells could only be detected in moderately differentiated regions of the tumours where a tissue architecture reminiscent of glandular epithelium was still maintained (Fig. 3A-C). The percentage of positive tumour cells, being highly variable also within a tumour type, did not significantly differ between the three tumour lines ( $\mathrm{p}=0.2$ and 0.4 ) (Fig. 4A). Sca-1 expressing cells could readily be detected in all nonmetastasising NeuT induced tumours, where they were regularly interspersed throughout the tumour tissue (Fig. 3D). Although not as frequently, single Sca-1 positive cells were also observed throughout the tissue of the majority of NeuT/ EphB4 and NeuT/truncated ephrin-B2 induced tumours. The quantification revealed that the frequency of Sca-1 positive cells did significantly differ between the NeuT induced tumours $(10 \%)$ and the tumours induced by NeuT/EphB4 $(1 \%)(\mathrm{p}=0.003)$ (Fig. 4B). Similarly, their frequency was significantly lower in the NeuT/truncated ephrin-B2 induced tumours $(4 \%)(\mathrm{p}=0.009)$. In both metastasising groups some tumour specimens were even completely negative for Sca-1 expressing cells (Fig. 4B).

CD24 expression is a characteristic for early but already restricted luminal progenitor cells in the mammary gland (39). In NeuT induced tumours, CD24 positive tumour cells 

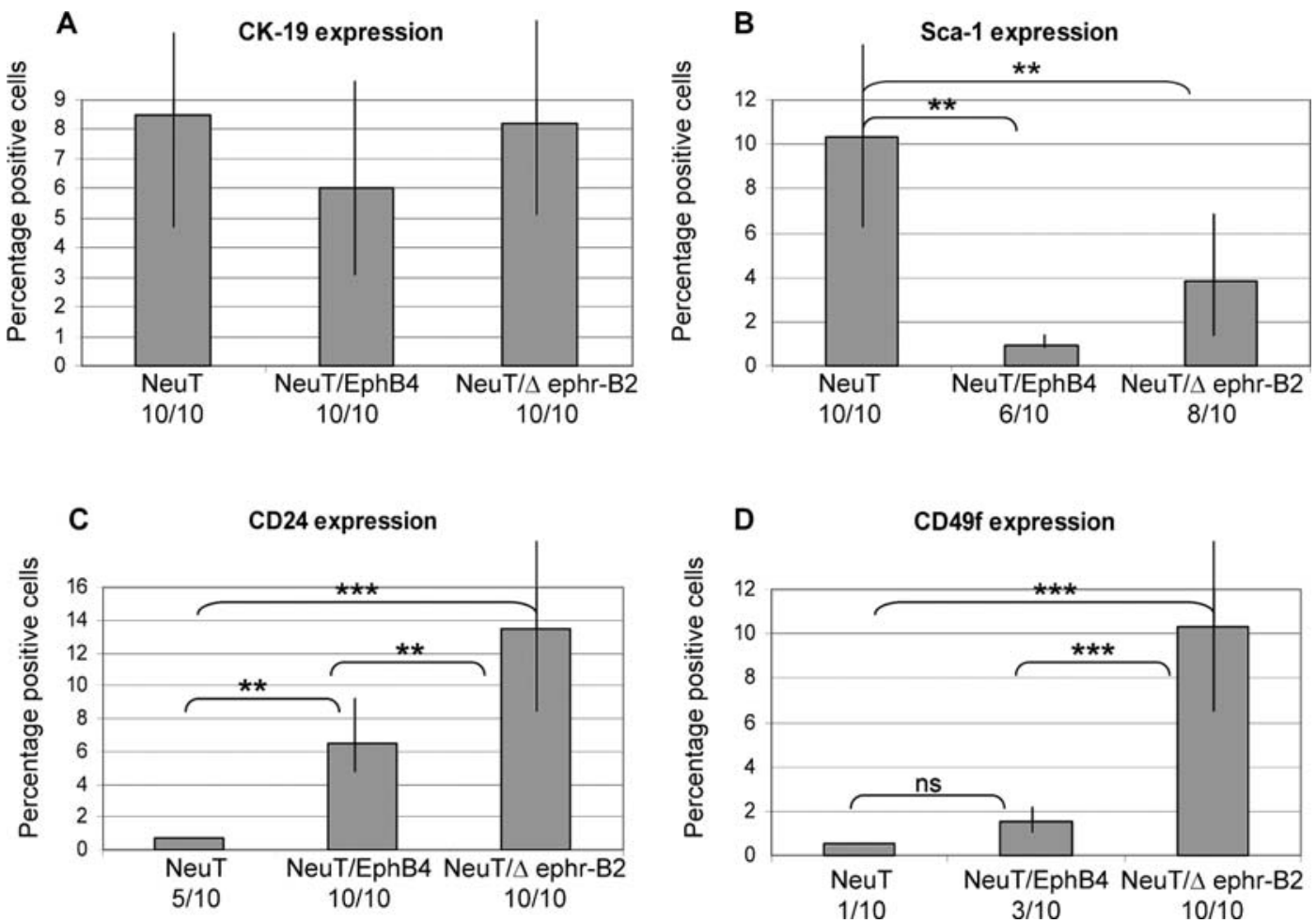

Figure 4. Quantification of cytokeratin-19 (CK19) (A), stem cell antigen-1 (Sca-1) (B), CD24 (C) and CD49f (D) expression in the NeuT, NeuT/EphB4 and NeuT/truncated ephrin-B2 (Lephrin-B2) induced tumours. Bars represent standard deviation. ns, statistically not significant; ${ }^{* *} \mathrm{p}<0.01,{ }^{* * *} \mathrm{p}<0.001$.
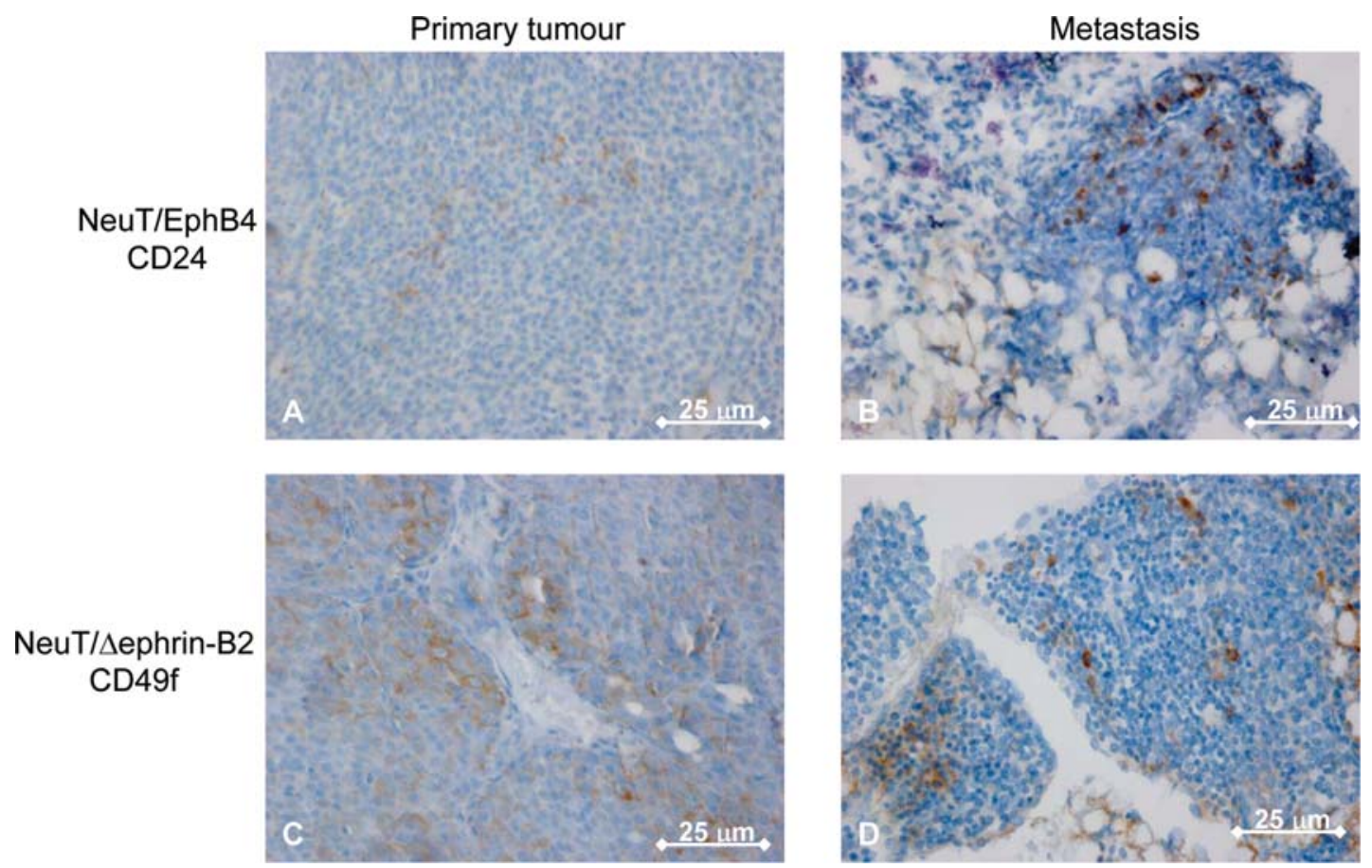

Figure 5. Expression of stem/progenitor cell markers in metastases. Immunohistochemical detection of CD24 expression in a primary tumour (A) and lung metastasis (B) induced by NeuT/EphB4 and of CD49f expression in a primary tumour (C) and lung metastasis (D) induced by NeuT/truncated ephrin-B2 (Dephrin-B2).

could rarely be found. Only a few cells were present in 5 out of 10 tumour samples. Prominent CD24 expression, however, was evident in the tumour vasculature (Fig. 3G). In contrast, nests of CD24 positive tumours cells were present in all specimens of both metastasising tumour types (Fig. 3H and I). Interestingly, in the NeuT/truncated ephrin-B2 tumours, the 


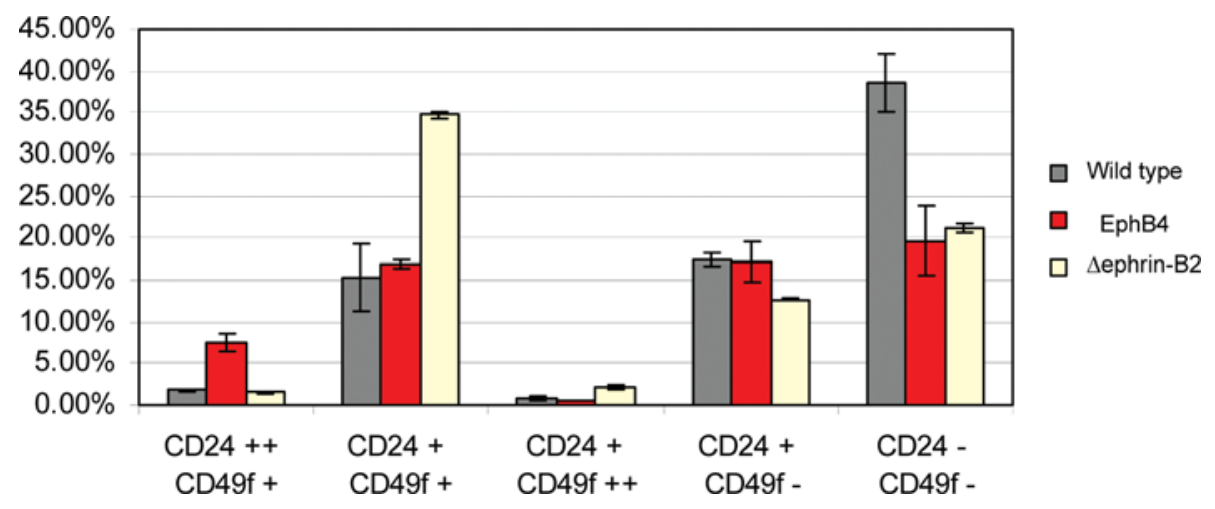

Figure 6. Quantification of stem/progenitor cell populations in control (wild-type) and the single transgenic MMTV-EphB4 (EphB4) and MMTV-truncated ephrin-B2 ( $\triangle$ ephrin-B2) animals by FACS-analysis. Cell populations were separated into CD24 high/CD49f low (CD24+ $/$ CD49f $)$, CD24 low/CD49f low $\left(\mathrm{CD} 24^{+} / \mathrm{CD} 49 \mathrm{f}^{+}\right), \mathrm{CD} 24$ low/CD49f high $\left(\mathrm{CD} 24^{+} / \mathrm{CD} 49 \mathrm{f}^{++}\right)$, CD24 low/CD49f negative (CD24 $\left./ \mathrm{CD} 49 \mathrm{f}^{\mathrm{f}}\right)$ and $\mathrm{CD} 24$ negative/CD49f negative $(\mathrm{CD} 24-\mathrm{CD} 49 \mathrm{f})$ cell populations. Bars represent the standard deviation, $\mathrm{N}=6$.

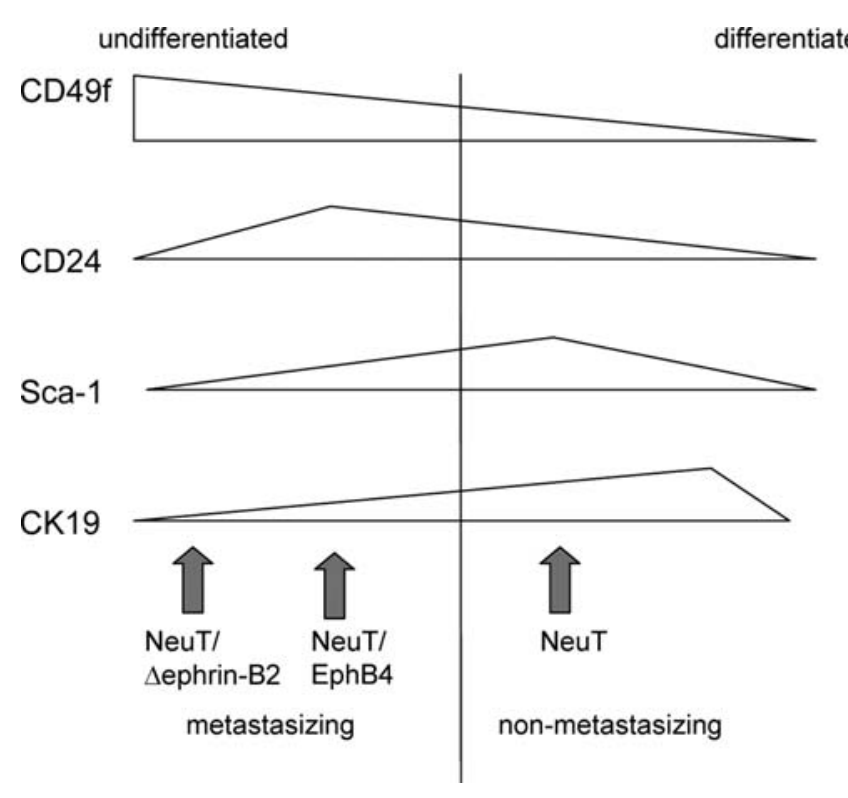

Figure 7. Schematic representation of the correlation between the differentiation grade of tumour initiating cells and metastasis formation.

intra-tumoural blood vessels were negative for CD24 expression (Fig. 3I). Although the amount of CD24 positive tumour cells was variable, a significant difference between the metastasising tumours $(6.3 \%$ for NeuT/EphB4 and $13.8 \%$ for NeuT/ truncated ephrin-B2) and the non-metastasising tumour type $(0.2 \%)$ was detected ( $\mathrm{p}=0.005$ and 0.0009 , resp.) (Fig. 4C)

High CD49f expression has been used to identify the unrestricted mammary epithelial stem cell able to differentiate in all cell types of the mammary parenchyma. Moreover, moderate CD49f expression was also seen in the bi-potent early progenitor cells (39). CD49f expressing tumour cells were virtually absent in the NeuT induced tumours and also extremely rare in the tumours induced by NeuT/EphB4 $(p=0.1)$. Again, the endothelium exhibited expression of CD49f (Fig. 3J and K). In contrast, small clusters of CD49f positive tumour cells interspersed throughout the tumour tissue could be detected in all specimens of NeuT/ephrin-B2 induced tumours (Fig. 3L). Their frequency differed significantly (at $10.2 \%$ ) from the low values found in the NeuT $(\mathrm{p}=0.0005)$ and NeuT/EphB4 $(\mathrm{p}=0.0007)$ tumour types (Fig. 4D).

These results indicate that the three tumour types differ in the presence of tumour progenitor cells. Whereas the Sca-1 positive cells are the least differentiated progenitor cells in the non-metastasising NeuT induced tumours, CD24 positive cells accumulated in the metastasising tumours of the NeuT/ EphB4 line. Metastasising NeuT/truncated ephrin-B2 tumours were further enriched in CD49f positive cells. If the CD24 and CD49f positive cells are capable of metastasis formation, these cells should also be present or even be enriched in the metastases. Indeed, compared to the primary tumours induced by NeuT/EphB4 the lung metastases of the same individuals contained an increased amount of cells strongly expressing the CD24 antigen (Fig. 5A and B). Similarly, CD49f positive cells accumulated in the lung metastases derived from NeuT/ truncated ephrin-b2 induced tumours (Fig. 5C and D).

These results suggest that deregulated EphB4-ephrin-B2 signalling may contribute to the acquisition of the metastasising phenotype by augmenting the stem- or early progenitor cell population. To investigate if this phenomenon is a direct consequence of this deregulated signalling or is only observed in combination with NeuT overexpression, we have quantified the CD24 and CD49f positive cells in the single transgenic EphB4 or truncated ephrin-B2 overexpressing animals by FACS analysis. The fourth inguinal mammary glands of adult resting control mice and MMTV-EphB4 or MMTV-truncated ephrin-B2 animals were dissociated and, after elimination of hematopoietic and endothelial cells, sorted according to the expression levels of CD24 and CD49f (Fig. 6). These experiments revealed that in the mammary glands of MMTV-EphB4 transgenic animals the CD $24^{++} / \mathrm{CD} 49 \mathrm{f}^{+}$cell population thought to harbour the luminal progenitor cells, is significantly increased. In contrast, the mammary epithelium of MMTVtruncated ephrin-B2 transgenic animals was significantly enriched in CD $49 \mathrm{f}^{++} / \mathrm{CD} 24^{+}$and in $\mathrm{CD} 49 \mathrm{f}^{+} / \mathrm{CD} 24^{+}$cells thought to contain the stem- and bi-potent progenitor cells, respectively. The enrichment of these cell populations was at the expense of CD49f/CD24- cells which contain the differentiated epithelium. This is in agreement with our previous morphological observations which indicated a disturbed 
epithelial differentiation in both transgenic lines $(18,19)$. These results demonstrate that deregulated EphB4 and ephrin-B2 expression directly results in the augmentation of the same stem/progenitor cell populations which were found to be enriched in the tumours of double-transgenic animals.

\section{Discussion}

According to the cancer stem cell hypothesis, tumour tissues exhibit a hierarchical tissue organisation similar to normal tissues. The bulk of tumour cells attain a certain level of differentiation which implies that these cells have a limited life span and are unable to reconstitute an entire new tumour. The hypothesis further claims that the tumour tissue also contains variable percentages of stem and progenitor cells which are immortal, maintain tumour growth and are able to initiated new tumour nodules even at distant locations (7). However, it is still under debate whether tumour growth originates from mutated organ stem cells or whether dedifferentiation of specialized tissue cells (also) can occur. Moreover, it has not been defined up to which differentiation level tumour cells are capable of forming metastases.

Our results contribute to the elucidation of these issues by the observation that metastasising tumours contain cells with characteristics of stem/progenitor cells with low differentiation grade which were absent in the non-metastasising tumours and thus must be responsible for metastasis formation. Moreover, our results suggest that tumour growth indeed had originated from the respective normal stem/progenitor cell population. Finally, we present evidence that the deregulated EphB4 and ephrin-B2 signalling confers a metastatic tumour phenotype by altering the mammary gland stem cell homeostasis.

The non-metastasising tumours induced by the NeuT oncogene were characterized by the presence of Sca- 1 positive cells. This is in agreement with a recent report defining the Sca-1 positive cells as the tumour initiating cells in this tumour model (40). Sca-1 expression is thought to define estrogen receptor (ER)-positive luminal epithelial cells. Although the main portion of this cell population is non-dividing and thus probably end-differentiated, dividing luminal restricted ERpositive progenitor cells are frequently found during puberty and are also present in adulthood (41). Moreover, Booth et al documented the presence of ER-positive cells in the adult mouse mammary gland exhibiting asymmetric cell division, a hall-mark of stem cells (42). Thus, it is likely that the expression of the NeuT oncogene is mainly affecting this ER-positive precursor cell population.

In contrast to the NeuT induced tumours, the metastasising NeuT/EphB4 or NeuT/truncated ephrin-B2 induced tumours were characterized by the presence of tumour cells exhibiting the expression of markers of a lower differentiation grade than Sca-1 expressing cells. CD24 expression defines the compartment enriched in luminal restricted progenitors which can differentiate into ER-positive or -negative luminal cells as well as into alveolar cells. In addition, bi-potent progenitors capable of differentiating into myo- or epithelial cells also express this surface marker in addition to CD49f (39). CD24 positive cells were detected in both metastasising tumour lines. The fact that NeuT/EphB4 induced tumours consistently contained CD24 positive cells at an average frequency of about $6 \%$, but almost no Sca-1 and CD49f expressing cells suggests that in this tumour model the CD24 positive luminal restricted precursors were the origin of carcinogenesis. CD24 is a glycosylphosphatidylinositol anchored membrane protein interacting with P-selectin and L1-CAM (43). Interestingly, a recent report suggests that $\mathrm{CD} 24$ is a negative regulator of side-branch formation during pubertal mammary epithelial morphogenesis (44). On the other hand CD24 has been identified as a marker for poor prognosis in a variety of cancer diseases including breast cancer (45) and CD24 expression promotes tumour growth and invasion in experimental mouse mammary tumours (46). It has been shown that CD24 can induce the localization of integrin- $\$ 1$ molecules to lipid rafts thereby influencing their functionality and stimulating migratory activity (47). Cellular migration is not only required for the evasion of precursor cells from their niche but may also contribute to the invasive phenotype of cancer cells. Moreover, through its capacity to bind to P-selectin CD24 may also contribute to metastasis formation by its ability to attach via P-selectin to endothelial cells which facilitates the extravasation of metastasising cells from the blood vessels.

The metastasising tumours induced by NeuT/truncated ephrin-B2 were the only tumour group which consistently contained CD49f positive tumour cells in the primary tumour and even enriched in the lung metastases. CD49f represents the integrin subunit $\alpha 6$ and together with its binding partners integrin- $\beta 1$ and $-\beta 4$ is the hallmark of the totipotent mammary stem cell and of the bi-potent mammary progenitor cells (39). High CD49f expression in breast cancer has been correlated with poor patient survival (48) and defines the xenograft initiating cells in triple negative and Her2 ${ }^{+} / \mathrm{ER}^{-}$breast cancer (49). Moreover, it has been shown that CD49f is involved in the homing process of breast carcinoma cells to the lung (50). CD49f has been shown to be involved in cell migration being localized at the leading edges of migrating cells (51). Interestingly, epithelial-mesenchymal transition, a hallmark of metastasising tumour cells, has been correlated with the reappearance of stem cell marker expression including CD49f (52). Thus, under normal conditions CD49f might be instrumental in the homeostasis of the stem cell niche and in the controlled evasion of stem cells thereof. During tumourigenesis CD49f may facilitate migration and the homing of metastatic cells to their target organs.

In addition to the expression of CD24 and CD49f, the metastasising tumours exhibited also a significant increase in cells expressing $\mathrm{p} 21^{\mathrm{waf}} \cdot \mathrm{p} 21^{\text {waf }}$ is a negative regulator of the cell cycle through its function to block DNA synthesis and to prevent cell cycle progression by inhibiting cyclin dependent kinases (38). Although its main function represents growth arrest to allow DNA repair, p2 ${ }^{\text {waf }}$ has also been implied in the attenuation of stem cell proliferation (53-55). In the metastasising tumours, p21 waf expressing cells were found in a comparable frequency as the cells positive for CD24 or CD49f. p21 waf expressing cells, however, were homogeneously interspersed throughout the tumour tissue, whereas the supposed tumour stem cells appeared mainly in clusters. This observation indicates that $\mathrm{p} 21^{\text {waf }}$ expression does not necessarily correlate with stem cell characteristics. Although $\mathrm{p} 21^{\text {waf }}$ has 
been classified as a tumour suppressor gene, it has become evident that the same molecule can also exert oncogenic functions either by its ability to prevent apoptosis and allow DNA repair or by preventing the nuclear export and degradation of cyclin-D $(56,57)$. Thus, it is conceivable that also in our system p21 $1^{\text {waf }}$ does not only mark attenuated precursor cells but also progressed cancer cells. The concomitant significant increase in the mitotic index indicative for an accelerated progression through the cell cycle would support this interpretation. Overexpression of $\mathrm{p} 21^{\text {waf }}$ has been correlated with cerbB2 expression and poor prognosis in breast cancer (38). In these cases, however, the poor prognostic value correlated with a cytoplasmic localization of $\mathrm{p} 21^{\text {waf }}$, whereas we have observed and evaluated its nuclear localization. Nevertheless, we can presently not define if the increase in p2 $1^{\text {waf }}$ expression reflects its oncogenic potential or its function in progenitor cell attenuation.

The NeuT/EphB4 induced tumours were characterized by the presence of CD24 positive cells, whereas the NeuT/ truncated ephrin-B2 induced tumours contained in addition a significant amount of CD49f expressing cells. Strikingly, these cell populations were also significantly increased in the mammary glands of single transgenic MMTV-EphB4 and MMTV-truncated ephrin-B2 females. This indicates that deregulated EphB4-ephrin-B2 signalling per se leads to a perturbation of the epithelial cell hierarchy in the mammary gland and to an accumulation of distinct progenitor/stem cell populations. A direct involvement of EphB receptors including EphB4 and ephrin-B ligands in the control of stem cell evasion and differentiation into progenitor cells has been shown for the intestine. Similar to our observation, wnt induced tumour formation led to an accumulation of EphB-positive progenitor cells which could only progress to adenocarcinomas after disruption of the proper EphB4-ephrin-B signalling (58). The mechanism(s) responsible for the observed effect of deregulated EphB4-ephrin-B2 signalling on the mammary stem/progenitor cell pool remain to be elucidated. It is of note, however, that the mammary epithelium of the single transgenic animals was characterized by a disturbed localization of E-cadherin and ß-catenin (17). Interestingly, the bipotent progenitor cells have been identified as tumour initiating cells also in mouse mammary tumours induced by the wnt oncogene (10). Moreover, it has been shown that mammary stem cells do not express E-cadherin and that silencing E-cadherin expression was sufficient for epithelial-mesenchymal transition and induction of a stem cell-like phenotype of mammary epithelial cells (59). Considering the crosstalk between EphB4, Ecadherin and the wnt- $\beta$-catenin pathways (60) and the importance of wnt- $\beta$-catenin signalling in the control of the mammary stem cell fate (10), it is conceivable that this route might be responsible for the observed effects.

Our observation that the same stem/progenitor cell populations are enriched in the normal tissue, primary mammary tumours and metastases allows the conclusion that these progenitor cells were the origin of tumour formation and that this change in the tumour origin has led to the acquisition of the metastatic tumour phenotype. The presence of cells exhibiting late progenitor characteristics such as Sca-1 or $\mathrm{ER} \alpha$ expression or of cells negative for all investigated markers support the notion that the majority of the tumour mass is composed of cells attaining a mixed degree of differentiation. Since these cells were also present in the nonmetastasising NeuT induced tumours they were not the cells responsible for metastasis formation. In summary, our results allow a classification of the metastatic potential of tumour initiating cells (Fig. 7). If undifferentiated stem cells as well as the bi-potent progenitor cells are the origin of malignant transformation, the resulting tumours are highly metastatic. This metastatic potential is lost as soon as the cells progress to the ER $\alpha$ and Sca-1-positive luminal precursor cells.

\section{Acknowledgements}

We wish to thank Robert Strange for helpful discussions and critical comments on the manuscript. This study was supported by the Swiss National Science Foundation (3100A0-109872), the Swiss Cancer League (KLS-02105-08-2007) and the Schweizerische Stiftung für Klinisch-Experimentelle Tumorforschung. The authors have no conflict of interest

\section{References}

1. Stockler M, Wilcken NR, Ghersi D and Simes RJ: Systematic reviews of chemotherapy and endocrine therapy in metastatic breast cancer. Cancer Treat Rev 26: 151-168, 2000

2. Micalizzi D, Farabaugh SM and Ford HL: Epithelialmesenchymal transition in cancer: parallels between normal development and tumour progression. J Mamm Gland Biol Neoplasia 15: 117-134, 2010.

3. Kaplan RN, Psaila B and Lyden D: Bone marrow cells in the 'pre-metastatic niche': within bone and beyond. Cancer Metastasis Rev 25: 521-529, 2006.

4. Allan AL, Vantyghem SA, Tuck AB and Chambers AF: Tumor dormancy and cancer stem cells: implications for the biology and treatment of breast cancer metastasis. Breast Dis 26: 87-98, 2007.

5. Appelbaum FR, Rowe JM, Radich J and Dick JE: Acute myeloid leukaemia. Hematology (Am Soc Hematol Educ Program) 2001: 62-86, 2001.

6. Al-Hajj M, Wicha MS, Benito-Hernandez A, Morrison SJ and Clarke MF: Prospective identification of tumorigenic breast cancer cells. Proc Natl Acad Sci USA 100: 3983-3988, 2003.

7. Zhou BB, Zhang H, Damelin M, Geles KG, Grindley JC and Dirks PB: Tumour initiating cells: challenges and opportunities for anticancer drug discovery. Nat Rev Drug Disc 8: 806-823, 2009.

8. Jordan CT, Guzman ML and Noble M: Cancer stem cells. N Engl J Med 355: 1253-1261, 2006.

9. Wicha MS, Liu S and Dontu G: Cancer stem cells: an old idea a paradigm shift. Cancer Res 66: 1883-1890, 2006.

10. Visvader JE: Keeping abreast of the mammary epithelial hierarchy and breast tumourigenesis. Genes Dev 23: 2563-2577, 2009.

11. Pasquale EB: Eph receptors and ephrins in cancer: bidirectional signalling and beyond. Nat Rev Cancer 10: 165-180, 2010.

12. Prevost N, Woulfe D, Tanaka T and Brass LF: Interactions between Eph kinases and ephrins provide a mechanism to support platelet aggregation once cell-cell contact has occurred. Proc Natl Acad Sci USA 99: 9214-9224, 2002.

13. Suenobu S, Takakura N, Inada T, Yamada Y, Yuasa H, Zhang XQ, Sakakano S, Oike Y and Suda T: A role of the EphB4 receptor and its ligand ephrin-B2 in erythropoiesis. Biochem Biophys Res Com 293: 1124-1131, 2002.

14. Okubo T, Yanai N and Obinata M: Stromal cells modulate ephrin-B2 expression and transmigration of hematopoietic cells. Exp Hematol 34: 330-338, 2006.

15. Zhao C, Irie N, Takada Y, Shimoda K, Miyamoto T, Nishiwaki T, Suda $\mathrm{T}$ and Matsuo K: Bidirectional signalling controls bone homeostasis. Cell Metab 4: 111-121, 2006.

16. Munarini N, Jäger R, Abderhalden S, Zürcher G, Rohrbach V, Lörcher S, Pfanner-Meyer B, Andres AC and Ziemiecki A: Altered mammary epithelial development, pattern formation and involution in transgenic mice expressing the EphB4 receptor tyrosine kinase. J Cell Sci 115: 25-37, 2002. 
17. Haldimann M, Custer D, Stirnimann C, Munarini N, Weiler S, Zürcher G, Rohrbach V, Djonov V, Ziemiecki A and Andres AC: Deregulated ephrin-B2 expression interferes with the development of both the glandular epithelium and vasculature and promotes metastasis formation. Int J Oncol 35: 525-536, 2009.

18. Battle E, Henderson JT, Benghte H, van den Born MWM, Sancho E, Huls G, et al: ß-catenin and Tcf mediate cell positioning in the intestinal epithelium by controlling the expression of EphB/ephrinB. Cell 111: 251-263, 2002.

19. Lazarova P, Wu Q, Kvalheim G, Suo Z, Haakenstad KW, Metodiev K and Nesland JM: Growth factor receptors in hematopoietic stem cells: Eph family expression in $\mathrm{CD}_{3} 4^{+}$and $\mathrm{CD}_{133^{+}}$cell populations from mobilised peripheral blood. Int J Immunopathol Pharmacol 19: 49-56, 2006.

20. Stokowski A, Shi S, Sun T, Bartold PM, Koblar SA and Gronthos S: EphB/ephrinB interaction mediates adult stem cell attachment, spreading and migration: implications for dental tissue repair. Stem Cells 25: 156-164, 2007.

21. Picco V, Hudson C and Yassuo H: Ephrin-Eph signalling drives the asymmetric division of notochord/neural precursors in Ciona embryos. Development 134: 1491-1497, 2007

22. Foubert P, Silvestre JS, Souttou B, Barateau V, Martin C, Ebrahimian TG, et al: PSGL-1 mediated activation of EphB4 increases the proangiogenic potential of endothelial progenitor cells. J Clin Invest 117: 1527-1537, 2007.

23. Pasquale EB: Eph-ephrin bidirectional signalling in physiology and disease. Cell 133: 38-52, 2008.

24. Takai N, Miyazaki T, Fujisawa K, Nasu K and Miyakawa I Expression of receptor tyrosine kinase EphB4 and its ligand ephrin-B2 is associated with malignant potential in endometrial cancer. Oncol Rep 8: 567-573, 2001.

25. Liu W, Ahmad SA, Jung YD, Reinmuth N, Fan F, Bucana CD, and Ellis LM: Coexpression of ephrin-Bs and their receptors in colon carcinoma. Cancer 94: 934-949, 2002.

26. Sawai Y, Tamura S, Fukui K, Ito N, Imanaka K, Saeki A, Sakuda S, Kiso S and Matsuzawa Y: Expression of ephrin-B1 in hepatocellular carcinoma: possible involvement in neovascularisation. J Hepatol 39: 991-996, 2003.

27. Berclaz G, Altermatt HJ, Rohrbach V, Flütsch B, Djonov V, Ziemiecki A, et al: Loss of EphB4 receptor tyrosine kinse protein expression during carcinogenesis of the human breast. Oncol Rep 9: 25-30, 2002.

28. Wu Q, Suo Z, Risberg B, Karlsson MG, Villman K and Nesland JM: Expression of EphB2 and EphB4 in breast carcinoma. Pathol Oncol Res 10: 26-33, 2004.

29. Noren NK, Lu M, Freeman AL, Koolpe M and Pasquale EB: Interplay between EphB4 on tumor cells and vascular ephrin-B2 regulates tumor growth. Proc Natl Acad Sci USA 101: 5583-5588, 2004 .

30. Noren NK, Foos G, Hauser CA and Pasquale EB: The EphB4 receptor suppresses breast cancer cell tumorigenicity through an Abl-Crk pathway. Nat Cell Biol 8: 815-825, 2006.

31. Martiny-Baron G, Korff T, Schaffner F, Esser N, Eggstein S, Marme D and Augustin HG: Inhibition of tumor growth and angiogenesis by soluble EphB4. Neoplasia 6: 248-257, 2004.

32. Kumar SR, Singh J, Xia G, Krasnoperov V, Hassanieh L, Ley EJ, et al: Receptor tyrosine kinase EphB4 is a survival factor in breast cancer. Am J Pathol 169: 279-293, 2006.

33. Masood R, Kumar SR, Sinha UK, Crowe DL, Krasnoperov V, Reddy RK, et al: EphB4 provides survival advantage to squamous cell carcinoma of head and neck. Int J Cancer 119: 1236-1248, 2006

34. Davalos V, Dopeso H, Castano J, Wilson AJ, Vilardell F, Romero-Gimenez J, et al: EphB4 and survival of colorectal cancer patients. Cancer Res 66: 8943-8948, 2006.

35. Kumar SR, Scehnet JS, Ley EJ, Krasnoperov V, Manchanda PK, Ladner RD, et al: Preferential induction of EphB4 over EphB2 and its implication in colorectal cancer. Cancer Res 69: 3736-3745, 2009

36. Dopeso H, Mateo-Lozano S, Mazzolini R, Rodrigues P, LagaresTena L, Ceron J, et al: The receptor tyrosine kinase EphB4 has tumour suppressor activities in intestinal tumorigenesis. Cancer Res 69: 7430-7438, 2009.

37. Nikolova Z, Djonov V, Zürcher G, Andres AC and Ziemiecki A Cell-type specific and estrogen dependent expression of the receptor tyrosine kinase EphB4 and its ligand ephrin-B2 during mammary gland morphogenesis. J Cell Sci 111: 2741-2751, 1998.
38. Abbas $\mathrm{T}$ and Dutta A: p21 in cancer: intricate network and multiple activities. Nat Rev Cancer 9: 400-414, 2009.

39. Stingl J: Detection and analysis of mammary stem cells. J Pathol 217: 229-241, 2009

40. Grange C, Lanzardo S, Cavallo F, Camussi G and Bussolati B: Sca-1 identifies the tumour-initiating cells in mammary tumours of balb-neuT transgenic mice. Neoplasia 10: 1433-1443, 2008.

41. Smith GH: Stem cells, hormones and mammary cancer. Adv Exp Med Biol 617: 69-78, 2008.

42. Booth BW and Smith GH: Estrogen receptor- $\alpha$ and progesterone receptor are expressed in label-retaining mammary epithelial cells that divide asymmetrically and retain their template DNA. Breast Cancer Res 8: R49, 2006.

43. Lim SC: CD24 and human carcinoma: tumour biological aspects. Biomed Pharmacother 59: 5351-5354, 2005.

44. Cremers N, Deugnier MA and Sleeman J: Loss of CD24 expression promotes ductal branching in the murine mammary gland. Cell Mol Life Sci 67: 2311-2322, 2010.

45. Kristiansen KJ, Winzer E, Mayordomo J, et al: CD24 expression is a new prognostic marker in breast cancer. Clin Cancer Res 9: 4906-4913, 2003.

46. Baumann P, Cremers N, Kroese F, Orend G, ChiquetEhrismann R, Uede T, Yagita H and Sleeman J: CD24 expression causes the acquisition of multiple cellular properties associated with tumour growth and metastasis. Cancer Res 65: 10783-10793, 2005.

47. Runz S, Mierke CT, Joumaa S, Behrens J, Fabry B and Altevogt P: CD24 induces localization of $\beta 1$ integrin to lipid rafts. Biochem Biophys Res Comm 365: 35-41, 2007.

48. Friedrichs K, Ruiz P, Franke F, Gille I, Terpe HJ and Imhof BA: High expression level of $\alpha 6$ integrin in human breast carcinoma is correlated with reduced survival. Cancer Res 55: 901-906, 1995.

49. Meyer MJ, Fleming JM, Lin AF and Hussnain A: CD44pos CD49f $\mathrm{f}^{\text {hi }} \mathrm{CD} 133 / 2^{\text {hi }}$ defines xenograft-initiating cells in estrogen receptor-negative breast cancer. Cancer Res 70: 4624-4633, 2010.

50. Mercurio AM, Bachelder RE, Chung J, O'Connor KL, Rabinovitz I, Shaw LM and Tani T: Integrin laminin receptors and breast carcinoma progression. J Mamm Gland Biol Neoplasia 6: 299-309, 2001

51. Rabinovitz I and Mercurio AM: The integrin $\alpha 634$ fiuctions in carcinoma cell migration on laminin-1 by mediating the formation and stabilization of actin-containing motility structures. J Cell Biol 139: 1873-1884, 1997.

52. Hollier BG, Evans K and Mani SA: The epithelial-mesenchymal transition and cancer stem cells: a coalition against cancer therapies. J Mamm Gland Biol Neoplasia 14: 29-43, 2009.

53. Topley GI, Okuyama R, Gonzales JG, Conti C and Dotto GP: p21 $1^{\text {waf/Cip } 1}$ functions as a suppressor of malignant skin tumour formation and a determinant of keratinocyte stem cell potential. Proc Natl Acad Sci USA 96: 9089-9094, 1999.

54. Cheng T, Rodrigues N, Shen H, Yarg Y, Dombkowski D, Skyes M and Scadden DT: Hematopietic stem cell quiescence maintained by p21 waf. Science 287: 1804-1808, 2000.

55. Kippin TE, Martens DJ and van der Kooy D: p21 loss compromises the relative quiescence of forebrain stem cell proliferation leading to exhaustion of their proliferative capacity. Genes Dev 19: 756-767, 2005.

56. Robinson IB: Oncogenic functions of tumour suppressor $\mathrm{p} 21^{\text {waf/Cip 1/Sdi1: }}$ association with cell senescence and tumourpromoting activities of stromal fibroblasts. Cancer Lett 179: 1-14, 2002.

57. Alt JR, Gladden AB and Diehl J: p21 Cip promotes cyclin D1 nuclear accumulation via direct inhibition of nuclear export. J Biol Chem 277: 8517-8523, 2002.

58. Merlos-Suarez A and Batlle E: Eph-ephrin signalling in adult tissues and cancer. Curr Opin Cell Biol 20: 194-200, 2008.

59. Morel AP, Lièvre M, Thomas C, Hinkal G, Ansieau S and Puisieux A: Generation of breast cancer stem cells through epithelial-mesenchymal transition. PLOS One 3: E2888, 2008.

60. Andres $\mathrm{AC}$ and Ziemiecki A: Eph and ephrin signalling in mammary gland morphogenesis and cancer. J Mamm Gland Biol Neopl 8: 475-486, 2003. 